\title{
Enumerating $\mathrm{A}_{3}(2)$ Blueprints, and an Application
}

\author{
Michael Abramson and Curtis D. Bennett
}

\section{CONTENTS}

\section{Definitions}

2. Enumerating Blueprints

3. Applications

References
We present an enumeration scheme for all blueprints of an $\mathrm{A}_{2}(\mathrm{p})$ building (with $\mathrm{p}$ prime). We then provide a computer based proof that the $\widetilde{A}_{n}\left(\mathbb{Q}_{2}\right)$ buildings do not conform to a blueprint for $\mathrm{n} \geq 3$.

J. Tits ([1955b; 1955a; 1956]; see the introduction of [Tits 1974] for a historical discussion) introduced buildings in an attempt to provide a systematic procedure for producing geometries associated to semisimple Lie groups. With this procedure, Tits found geometries associated to the exceptional groups $E_{6}, E_{7}$, and $E_{8}$. One of the early hopes for buildings was to provide a way to combinatorially construct the finite groups of Lie type, and in [Ronan and Tits 1987], this was accomplished using blueprints. Ronan and Tits, however, were unable to find blueprints constructing general $p$ adic affine buildings. At the end of their paper though, they suggested a generalization of a blueprint which might be used.

Abramson $[\geq 1998]$ presented a generalization of a blueprint like that suggested by Ronan and Tits. He used this to construct the $p$-adic buildings. The question of whether in general $p$-adic buildings conform to blueprints was left unanswered.

Currently there is only one known case of a $p$ adic building with a blueprint. Ronan ([1984]; see [Kantor 1986] for a complete discussion) presented four buildings of type $\widetilde{A}_{2}$ associated to the Frobenius group of order 21. P. Köhler, T. Meixner, and M. Wester proved that one of these corresponded to the classical $\widetilde{A}_{2}$ building associated to the field $\mathbb{F}_{2}((t))$ [Köhler et al. 1984] and a second corresponded to the 2 -adic building of type $\widetilde{A}_{2}$ over the 
field $\mathbb{Q}_{2}$ [Köhler et al. 1985]. These presentations lead naturally to blueprints.

Here we show, using computer evidence, that most $\mathbb{Q}_{2}$ buildings do not conform to blueprints. To do this we first prove:

Theorem 1. The only blueprints for the $A_{3}(2)$ building are relabelings of the natural blueprint.

From this we obtain:

Theorem 2. The simply-laced affine buildings of rank at least 4 (with connected diagram) over $\mathbb{Q}_{2}$ do not conform to blueprints.

\section{DEFINITIONS}

A chamber system $\left(\mathcal{C},\left\{P_{i}\right\}_{i \in I}\right)$ is a set $\mathcal{C}$ of chambers and a set $\left\{P_{i}\right\}_{i \in I}$ of partitions of $\mathcal{C}$. We say that two chambers $C \neq D$ of $\mathcal{C}$ are $i$-adjacent if $C$ and $D$ lie in the same part of the partition $P_{i}$, and we write $C \sim D$. A gallery from $C$ to $D$ is a sequence of chambers $C=C_{0}, C_{1}, \ldots, C_{n}=D$, such that $C_{j-1} \underset{i_{j}}{\sim} C_{j}$ for $j=1, \ldots, n$. The type of the gallery $C_{0}^{i_{j}} \underset{i_{1}}{ } C_{1} \underset{i_{2}}{ } \ldots \underset{i_{n}}{\widetilde{i_{n}}} C_{n}$ is the word $i_{1} i_{2} \ldots i_{n}$, and its length is $n$. A gallery from $C$ to $D$ is minimal if there exists no shorter gallery from $C$ to $D$.

Let $M=\left(m_{i j}\right)_{i, j \in I}$ be a symmetric integer matrix with diagonal entries 1 having $m_{i j} \geq 2$ for $i \neq j$. The associated Coxeter diagram is a graph whose vertex set is labeled by the elements of $I$ and whose edge set consists of edges labeled $m_{i j}$ for all $m_{i j} \geq 3$. The diagram is simply-laced if $m_{i j} \leq 3$ for all $i$ and $j$.

Given $M$ as above let $W$ be the Coxeter group

$$
W=\left\langle r_{i} \mid i \in I, r_{i}^{2}=\left(r_{i} r_{j}\right)^{m_{i j}}=1\right\rangle .
$$

We say that $W$ is spherical if $|W|<\infty$ and the rank of $W$ is $|I|$. For $w \in W$ define the length, $l(w)$, to be the least $k$ such that $w=r_{i_{1}} \ldots r_{i_{k}}$. Given a word $f=i_{1} i_{2} \ldots i_{n}$ define $r_{f}=r_{i_{1}} \ldots r_{i_{n}}$. The word $f$ is reduced if $l\left(r_{f}\right)=n$.
Let $W$ be a Coxeter group with index set $I$. A chamber system $\left(\mathcal{C},\left\{P_{i}\right\}_{i \in I}\right)$ is a building of type $W$ if there exists a $W$-distance function

$$
\delta: \mathcal{C} \times \mathcal{C} \rightarrow W
$$

such that for all reduced words $f$ there exists a gallery of type $f$ from $C$ to $D$ if and only if we have $\delta(C, D)=r_{f}$ [Tits 1981].

Let $W$ be a Coxeter group with index set $I$, and let $S_{i}$ be an index set for each $i \in I$. A labeled word of type $f$ is a reduced word $f=i_{1} i_{2} \ldots i_{t}$ together with a sequence $a=\left(a_{1}, \ldots, a_{t}\right)$ where $a_{t} \in S_{i_{t}}$ for all $t$. We write this as

$$
f^{a}=i_{1}^{a_{1}} i_{2}^{a_{2}} \ldots i_{t}^{a_{t}} .
$$

Define a chamber complex with $\mathcal{C}$ the set of labeled words and $i$-adjacency given by

$$
f^{a} i^{b} \underset{i}{\sim} f^{a} i^{c} \underset{i}{\sim} f^{a}
$$

where $f i$ is reduced, $a$ is a label for $f$, and $b, c \in S_{i}$. Given a word $f$, define $\mathcal{C}_{f}=\left\{f^{a} \in \mathcal{C}\right\}$.

Given $i$ and $j$, let $p(i, j)=\ldots i j$ be the word of length $m_{i j}$. If $I=\{i, j\}$ a rank 2 equivalence for $\mathcal{C}$ is a bijection $\varphi: \mathcal{C}_{p(i, j)} \rightarrow \mathcal{C}_{p(j, i)}$. Given a rank 2 equivalence $\varphi$, define $\mathrm{C}(\varphi)$ to be the chamber complex obtained by identifying $p(i, j)^{a}$ with $\varphi\left(p(i, j)^{a}\right)$ where for ease of notation, we write $p(i, j)^{\varphi(a)}$ for the latter. More precisely, the chambers of $\mathcal{C}(\varphi)$ are the equivalence classes of chambers of $\mathcal{C}$ where two equivalence classes are $i$-adjacent if they contain $i$-adjacent elements. The rank 2 equivalence $\varphi$ is a rank 2 blueprint if $\mathcal{C}(\varphi)$ is a building of type $W$.

Example A. Consider the case where $m_{12}=2$ and $\varphi\left(1^{a} 2^{b}\right)=2^{b} 1^{a}$. Then we have $1^{a} 2^{b} \sim 2^{b} 1^{c}$ for all $a, b, c$ in the appropriate label sets. In this case $\varphi$ is a rank 2 blueprint.

Example B. Alternatively, if $\varphi$ is defined by $\varphi\left(i^{a} j^{b}\right)=$ $j^{a} i^{b}$ for all labels $a$ and $b$, then $1^{a} 2^{b} \sim 2^{a} 1^{c}$ for all $c$, and $\varphi$ is not a rank 2 blueprint since the shortest gallery from $1^{a}$ to $2^{b} 1^{c}$, is of length 3 when $a \neq b$. 
Given $W, I, S_{i}$, and $C$ as above, a blueprint for $\mathcal{C}$ is the collection

$$
\mathcal{B}=\left\{\varphi_{i j}: \mathcal{C}_{p(i, j)} \rightarrow \mathcal{C}_{p(j, i)} \mid i \neq j \in I\right\}
$$

satisfying $\varphi_{i j}=\varphi_{j i}^{-1}$ and such that $\varphi_{i j}$ is a rank 2 blueprint for all 2-element subsets $\{i, j\}$ of $I$.

Given a blueprint $\mathcal{B}$, define the labeled words $h^{a} p(j, i)^{b} g^{c}$ and $h^{a} p(i, j)^{\varphi_{i j}(b)} g^{c}$ to be elementarily $\mathcal{B}$-equivalent. Let $\sim$ be the transitive closure of this relation, and define $\mathcal{C}(\mathcal{B})=\mathcal{C} / \sim$; that is, the chambers of $\mathcal{C}(\mathcal{B})$ are the equivalence classes of chambers of $\mathcal{C}$ with two equivalence classes $i$-adjacent if they contain elements which are $i$-adjacent in $\mathrm{C}$. Following the notation of [Ronan and Tits 1987], we define a blueprint to be realizable if $\mathcal{C}(\mathcal{B})$ is a building. Conversely, given a building $\Delta=\mathfrak{C}(\mathcal{B})$, we say that $\Delta$ conforms to the blueprint $\mathcal{B}$.

Associated to any Coxeter group with index set $I$ is a diagram whose vertices correspond to the elements of $I$ and where the vertices $i$ and $j$ are connected by $0,1,2$, or 3 bonds, as the order of $r_{i} r_{j}$ is two, three, four, or six respectively. If the order of $r_{i} r_{j}$ is some other integer $n$, then we represent this on the diagram by joining vertices $i$ and $j$ by a bond with the label $n$.

The following special case of [Ronan and Tits 1987, Theorem 1] simplifies checking whether a blueprint is realizable.

Theorem 3 [Ronan and Tits 1987]. A blueprint $\mathcal{B}$ associated to a simply-laced diagram is realizable if and only if its restriction to the following subdiagrams is also realizable:

1. $A_{1} \times A_{1} \times A_{1}: \quad \circ \circ \circ$

2. $A_{1} \times A_{2}: \quad \circ \bigcirc$

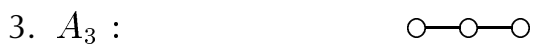

Moreover in this case there is a unique building $\Delta$ conforming to $\mathcal{B}$.

The natural blueprint of type $A_{2}(p)$ ( $p$ a prime) is the rank 2 blueprint given by

$$
\varphi\left(1^{a} 2^{b} 1^{c}\right)=2^{c} 1^{a c-b} 2^{a}
$$

where $a, b, c \in \mathbb{F}_{p}$.

The natural blueprint of type $\left(A_{1} \times A_{1}\right)(p)$ is given by

$$
\varphi\left(1^{a} 2^{b}\right)=2^{b} 1^{a}
$$

where $a, b \in \mathbb{F}_{p}$.

\section{ENUMERATING BLUEPRINTS}

In [Ronan and Tits 1987] natural blueprints are used to construct geometries associated to the Chevalley groups and from this the Chevalley groups themselves. Other blueprints may also give rise to interesting groups.

For example, the Frobenius group of 21 elements gives rise to $A_{2}(2)$ blueprints (not natural) which can be used to construct a blueprint for the $\widetilde{A}_{2}\left(\mathbb{Q}_{2}\right)$ building in the following fashion. Define $\gamma: \mathbb{Z}_{2}^{3} \rightarrow$ $\mathbb{Z}_{2}^{3}$ by

$$
\begin{array}{ll}
\gamma(0,0,0)=(1,0,0), & \gamma(1,0,0)=(0,1,1), \\
\gamma(0,0,1)=(0,0,0), & \gamma(1,0,1)=(1,0,1), \\
\gamma(0,1,0)=(0,1,0), & \gamma(1,1,0)=(0,0,1), \\
\gamma(0,1,1)=(1,1,1), & \gamma(1,1,1)=(1,1,0) .
\end{array}
$$

Now define $\varphi_{1,2}$ by $\varphi_{1,2}\left(1^{a} 2^{b} 1^{c}\right)=1^{x} 2^{y} 1^{z}$ where $\gamma(a, b, c)=(x, y, z)$. Define $\varphi_{2,3}$ and $\varphi_{3,1}$ analogously, and then $\varphi_{2,1}, \varphi_{3,2}$, and $\varphi_{1,3}$ are then the respective inverses. By [Tits 1990, Remark 2], this is then a blueprint for the affine building of $\mathrm{SL}_{3}\left(\mathbb{Q}_{2}\right)$.

In this paper, our goal is to provide a starting point for answering the question of what buildings conform to blueprints. In particular, we will show that no buildings over $\mathbb{Q}_{2}$ with triangle-free simplylaced diagrams conform to a blueprint.

By Theorem 3, a blueprint is realizable if and only if it is realizable on the appropriate subdiagrams. As a result our strategy is to enumerate all rank 2 blueprints, and then check when three such blueprints combine to form a realizable blueprint.

Let $I=\{1,2\}$, and let $S_{1}=S_{2}=\{0, \ldots, n\}$. Given a non-empty word $f=\ldots i$, let $\Sigma_{f}$ denote 
the symmetric group on $S_{j}$ where $j \neq i$. Define $X$ to be the set

$$
X=\operatorname{Sym}\left(S_{1}\right) \times \operatorname{Sym}\left(S_{2}\right) \times \prod_{\substack{f^{a} \in \mathcal{C} \\ l(f)<m_{1,2}}} \Sigma_{f} .
$$

If $m_{1,2}=3$, an arbitrary element of $X$ has the form

$$
\begin{aligned}
\sigma=( & \sigma_{1}, \sigma_{2}, \sigma_{1^{0}}, \ldots, \sigma_{1^{n}}, \sigma_{2^{1}}, \ldots, \sigma_{2^{n}}, \\
& \sigma_{1^{0} 2^{0}}, \ldots, \sigma_{1^{0} 2^{n}}, \sigma_{1^{1} 2^{0}}, \ldots, \sigma_{1^{n} 2^{n}}, \\
& \left.\sigma_{2^{0} 1^{0}}, \sigma_{2^{0} 1^{1}}, \ldots, \sigma_{2^{n} 1^{n}}\right) .
\end{aligned}
$$

Let $W$ be a spherical Coxeter group of rank 2, and suppose $\left\{S_{1}, S_{2}\right\}$ is a labeling. Then for $\sigma \in X$ as above, define a bijection $\psi_{\sigma}$ on $\mathcal{C}$ by

$$
\psi_{\sigma}\left(i^{a} j^{b} \ldots\right)=i^{\sigma_{i}(a)} j^{\sigma_{i}^{a}(b)} \ldots
$$

The set $\Psi=\left\{\psi_{\sigma} \mid \sigma \in X\right\}$ is then a group under composition. Given a rank 2 blueprint $\varphi$, define $\psi_{\sigma}(\varphi)=\varphi^{\sigma}$ by

$$
\varphi^{\sigma}\left(f^{a}\right)=\psi_{\sigma}\left(\varphi\left(\psi_{\sigma}^{-1}\left(f^{a}\right)\right)\right)
$$

where $f$ is any reduced word of length $m_{i, j}$. This defines an action of $G$ on the set $\left\{\psi_{\sigma}(\varphi) \mid \psi_{\sigma} \in G\right\}$.

Lemma 4. With $W, S_{1}, S_{2}, \mathcal{C}, \sigma, \psi_{\sigma}$, and $\varphi$ as above, the map $\varphi^{\sigma}$ is a rank 2 blueprint for $\mathcal{C}$. Moreover, $\psi_{\sigma}$ induces an isomorphism from $\mathrm{C}(\varphi)$ to $\mathrm{C}\left(\varphi^{\sigma}\right)$.

Proof. We first show that $\psi_{\sigma}$ maps equivalent elements of $\mathcal{C}(\varphi)$ to equivalent elements of $\mathcal{C}\left(\varphi^{\sigma}\right)$. If the equivalence class has only one element, there is nothing to check. Else, suppose $f^{a} \sim h^{b}$ in $\mathcal{C}(\varphi)$. Then $l(f)=m_{1,2}$ and $\varphi\left(f^{a}\right)=h^{b}$. By definition,

$$
\varphi^{\sigma}\left(\psi_{\sigma}\left(f^{a}\right)\right)=\psi_{\sigma}\left(\varphi\left(f^{a}\right)\right)=\psi_{\sigma}\left(h^{b}\right) .
$$

Since $\psi_{\sigma}$ maps $i$-adjacent elements of $\mathcal{C}(\varphi)$ to $i$ adjacent elements of $\mathcal{C}\left(\varphi^{\sigma}\right)$, it follows that $\psi_{\sigma}$ induces an isomorphism of these two chamber systems. If $\varphi$ is a rank 2 blueprint then $\mathcal{C}(\varphi)$ is a building and hence so is $\mathcal{C}\left(\varphi^{\sigma}\right)$.

Lemma 5. Given $W, S_{1}, S_{2}, \mathcal{C}, \Psi$, as above, if $\varphi$ and $\gamma$ are two rank 2 blueprints and $\alpha: \mathcal{C}(\varphi) \rightarrow \mathcal{C}(\gamma)$ is a type preserving isomorphism of chamber complexes fixing the base chamber, (the trivial labeled word $\varnothing)$, then $\gamma=\varphi^{\sigma}$ for some $\psi_{\sigma} \in \Psi$ completely determined by $\alpha$. In particular, if $\alpha: \mathcal{C}(\varphi) \rightarrow$ $\mathcal{C}(\varphi)$, then $\alpha=\psi_{\sigma}$ for some $\sigma \in X$ with $\varphi^{\sigma}=\varphi$.

Proof. Let $\alpha$ be as above. Then $\alpha$ lifts to an isomorphism $\hat{\alpha}$ on $\mathcal{C}$ defined by $\hat{\alpha}\left(f^{a}\right)=f^{b}$ where $\alpha\left(\left[f^{a}\right]_{\varphi}\right)=\left[f^{b}\right]_{\gamma}$, where $\left[f^{a}\right]_{\varphi}$ denotes the equivalence class of $f^{a}$ in $\mathrm{C}(\varphi)$; hence given $a_{j} \in S_{i_{j}}$, there exist $b_{j} \in S_{i_{j}}$ such that

$$
\hat{\alpha}\left(i_{1}^{a_{1}} i_{2}^{a_{2}} \ldots\right)=i_{1}^{b_{1}} i_{2}^{b_{2}} \ldots,
$$

and we have $\gamma \circ \hat{\alpha}=\hat{\alpha} \circ \varphi$. Let $\sigma_{1}$ be the permutation of $S_{1}$ such that $1^{\sigma_{1}(a)}=\hat{\alpha}\left(1^{a}\right)$, and let $\sigma_{2}$ be the permutation of $S_{2}$ such that $2^{\sigma_{2}(a)}=\hat{\alpha}\left(2^{a}\right)$. Given $\sigma_{1}(a)=b$, define $\sigma_{1^{a}}$ by $1^{b} 2^{\sigma_{1^{a}(c)}}=\hat{\alpha}\left(1^{a} 2^{c}\right)$. Continue inductively so that if $\hat{\alpha}\left(f^{a}\right)=f^{b}$ then $\sigma_{f^{a}}$ is defined by $\hat{\alpha}\left(f^{a} i^{c}\right)=f^{b} i^{\sigma_{f^{a}}(c)}$, and let $\sigma \in X$ be the element with first coordinate $\sigma_{1}$, second coordinate $\sigma_{2}$ and $f^{a}$ th coordinate $\sigma_{f^{a}}$. By the definition of $\hat{\alpha}$, we have $\hat{\alpha}\left(\varphi\left(1^{a} 2^{b} 1^{c}\right)\right)=\gamma\left(\hat{\alpha}\left(1^{a} 2^{b} 1^{c}\right)\right)$. By the definition of $\sigma$, we have $\alpha=\psi_{\sigma}$. Hence $\gamma=\varphi^{\sigma}$.

What Lemmas 4 and 5 imply is that given a rank 2 building $\Delta$ and one blueprint, $\varphi$, realizing it, every other blueprint realizing $\Delta$ is of the form $\varphi^{\sigma}$ for some $\sigma \in X$. Moreover, if there is a non-trivial automorphism of the rank 2 building fixing the base chamber then this automorphism corresponds to an element $\sigma \in X$ such that $\varphi^{\sigma}=\varphi$. Similarly, given an element $\sigma \in X$ such that $\varphi^{\sigma}=\varphi$, the map $\psi_{\sigma}$ must be an automorphism of the building fixing $\varnothing$. Let $H$ be the stabilizer in $\Psi$ of the blueprint $\varphi$ under the action $\psi_{\sigma}(\varphi)=\varphi^{\sigma}$. Then an efficient enumeration scheme for blueprints realizing $\mathrm{C}(\varphi)$ would pick out exactly one element of each coset of $H$.

Theorem 6. Let $\Delta$ be a rank 2 building with $m_{1,2}=3$ and parameter $p$ a prime. Suppose $\left(\left\{S_{1}, S_{2}\right\}, \varphi\right)$ is a rank 2 blueprint realizing $\Delta$. Then every blueprint realizing $\Delta$ is given by $\varphi^{\sigma}$, where

$$
\sigma=\left(\sigma_{1}, \ldots\right) \in X
$$


can be chosen uniquely so that

$$
\begin{aligned}
& \sigma_{1}(0)=\sigma_{1^{0}}(0)=\sigma_{1^{0} 2^{0}}(0)=0, \\
& \sigma_{1}(1)=\sigma_{1^{0}}(1)=1 .
\end{aligned}
$$

Proof. By Lemma 5 every blueprint realizing $\Delta$ is of the form $\varphi^{\sigma}$ for some $\sigma$. It remains to show that $\sigma$ can be uniquely chosen satisfying equations $(2-1)$. Suppose $\sigma$ satisfies these equations and $\varphi^{\sigma}=\varphi$. Then the map $\psi_{\sigma}$ fixes the apartment of $\mathcal{C}(\varphi)$ containing the chambers $[\varnothing],\left[1^{0}\right],\left[1^{0} 2^{0}\right],\left[1^{0} 2^{0} 1^{0}\right],\left[1^{1}\right]$, and $\left[1^{0} 2^{1}\right]$. Moreover, since this is the $A_{2}(p)$ building and $\psi_{\sigma}$ fixes $\left[1^{1}\right]$ and $\left[1^{0} 2^{1}\right]$, then $\psi_{\sigma}$ fixes every chamber of form $\left[1^{a}\right]$ and every chamber of form $\left[1^{0} 2^{a}\right]$. By [Tits 1974, Theorem 4.1.1], this implies that $\psi_{\sigma}$ is the identity on $\Delta$. In fact, the requirement that $\psi_{\sigma}$ fixes the given apartment and the base chamber implies $\psi_{\sigma}$ is a diagonal matrix under the appropriate basis for $\mathbb{F}_{p}^{3}$. The requirements that $\left[1^{1}\right]$ and $\left[1^{0} 2^{1}\right]$ are fixed imply that $\psi_{\sigma}$ corresponds to the matrix $k I$ for some $k \in \mathbb{F}_{p}$. But then $\psi_{g}$ acts as the identity on $\Delta$. As a result, if $\gamma$ is any blueprint for $\Delta$, then there exists at most one element $\sigma \in X$ satisfying equations (2-1) with $\gamma=\varphi^{\sigma}$.

Alternatively, if $\gamma$ is a blueprint, Lemma 5 implies that $\gamma=\psi_{\sigma}(\varphi)$ for some $\sigma \in X$. Since $\mathrm{PSL}_{3}(p)$ is transitive on apartments of $\mathcal{C}(\varphi)$ (which follows from [Ronan 1989, Theorem 5.3]), there exists an automorphism $\alpha$ such that $\alpha(\varnothing)=\varnothing$ and $\alpha\left(\psi_{\sigma}\left(1^{0} 2^{0} 1^{0}\right)\right)=1^{0} 2^{0} 1^{0}$. Since the stabilizer of an apartment is transitive on ordered pairs of chambers of the form $\left(\left[1^{a}\right],\left[1^{0} 2^{a}\right]\right)$, with $a, b \neq 0$, we can choose $\alpha$ such that $\alpha(\varnothing)=\varnothing, \alpha\left(\psi_{\sigma}\left(1^{0} 2^{0} 1^{0}\right)\right)=$ $1^{0} 2^{0} 1^{0}, \alpha\left(\psi_{\sigma}\left(1^{1}\right)\right)=1^{1}$, and $\alpha\left(\psi_{\sigma}\left(1^{0} 2^{1}\right)\right)=1^{0} 2^{1}$. By Lemma $5, \alpha=\psi_{\tau}$ for some $\tau \in X$ and $\psi_{\tau}(\gamma)=$ $\gamma$. Since $\Psi$ is a group, $\psi_{\tau} \circ \psi_{\sigma}=\psi_{\eta}$ for some $\eta \in X$. Hence $\gamma=\varphi^{\eta}$ where $\eta$ satisfies $(2-1)$.

Since there exist efficient algorithms for producing permutations (in [Rosen 1991, p. 284], for example), this theorem together with such an algorithm provides a way of enumerating all the blueprints of $A_{2}(p)$, where $p$ is a prime. The following corol- lary provides a count for the number of $A_{2}(p)$ blueprints:

Corollary 7. The number of blueprints for the $A_{2}(p)$ building is

$$
\frac{(p !)^{\left(2 p^{2}+2 p+2\right)}}{p^{3}(p-1)^{2}} .
$$

Proof. The number of blueprints is clearly $|\Psi| /|H|$, where $H$ is the stabilizer of the natural blueprint. Above, we proved that the stabilizer of $H$ has order $p^{3}(p-1)^{2}$.

For $p=2$ this number is 2048 , for $p=3$ the number is approximately $1.57 \times 10^{18}$. As a result, pure enumeration schemes are only reasonable for the $p=2$ case.

One can generalize Theorem 6 to other buildings, but the results are not as easily stated. For this paper, we also need the $\left(A_{1} \times A_{1}\right)(p)$ case. In this case the building corresponds to the complete bipartite graph $K_{p+1, p+1}$, and the stabilizer of a chamber in the automorphism group is isomorphic to $S_{p} \times S_{p}$. The number of blueprints is $(p !)^{2 p}$ giving 16 blueprints for $p=2$.

We now turn to the question of blueprints for higher-rank buildings. If we are given a blueprint $\mathcal{B}$ for the building $\Delta$, then corresponding to any element of $\coprod_{i} \operatorname{Sym}\left(S_{i}\right)$ we get a blueprint simply by permuting the elements of $S_{i}$, that is by mapping $i_{1}^{a_{1}} \ldots i_{k}^{a_{k}}$ to the element $i_{1}^{\tau_{i_{1}}\left(a_{1}\right)} \ldots i_{k}^{\tau_{i_{k}}\left(a_{k}\right)}$. In general, this blueprint will not be equal to the original blueprint, although in the natural $A_{1} \times A_{1}$ case it is. Consider the case of the natural blueprint $\varphi$ for $A_{2}(p)$, and let $\sigma \in \operatorname{Sym}\left(S_{1}\right)$ and $\tau \in \operatorname{Sym}\left(S_{2}\right)$. Since

$$
\varphi\left(1^{a} 2^{b} 1^{c}\right)=2^{c} 1^{a c-b} 2^{a},
$$

for $(\sigma, \tau)$ to fix $\varphi$ would require $\sigma(a)=\tau(a)$ for all $a \in S_{i}$, and hence that $\sigma=\tau$. Moreover, we would have $\sigma(a c-b)=\sigma(a) \sigma(c)-\sigma(b)$ for all $a, b, c \in$ $S_{1}=S_{2}$, implying $\sigma$ is a field automorphism of $\mathbb{F}_{p}$. Hence $\sigma$ is trivial. We have shown:

Theorem 8. There are $(p !)^{3}$ distinct relabelings of the natural $A_{3}(p)$ blueprint. 


\section{APPLICATIONS}

In this section we summarize results from computer analysis on $A_{3}(2)$-blueprints. All programs were run using MATLAB on a Silicon Graphics Indy computer. To find all $A_{3}(2)$ blueprints, we enumerated the rank 2 blueprints of type $A_{2}(2)$ and $A_{1}(2) \times A_{1}(2)$. We then checked all triples $\left(\varphi_{1,2}, \varphi_{2,3}, \varphi_{1,3}\right)$, where $\varphi_{1,2}$ and $\varphi_{2,3}$ are $A_{2}(2)$ blueprints and $\varphi_{1,3}$ is an $\left(A_{1} \times A_{1}\right)(2)$ blueprint. These maps then produce a blueprint $\mathcal{B}$. To see if this blueprint realizes the $A_{3}(2)$ building, by [Ronan 1989, Theorem 7.2] it suffices to check that if $f^{a}$ is equivalent to $f^{b}$ in $\mathcal{C}(\mathcal{B})$ then $a=b$. In turn to check this it suffices as in [Ronan 1989, p. 93] to see that the following self-homotopy induces a self-equivalence:

$$
\begin{aligned}
1^{a} 2^{b} 1^{c} 3^{d} 2^{e} 1^{f} \sim & 2^{a_{1}} 1^{b_{1}} 2^{c_{1}} 3^{d} 2^{e} 1^{f} \\
& \left(\text { where } \varphi_{12}\left(1^{a} 2^{b} 1^{c}\right)=2^{a_{1}} 1^{b_{1}} 2^{c_{1}}\right) \\
\sim & 2^{a_{1}} 1^{b_{1}} 3^{c_{2}} 2^{d_{1}} 3^{e_{1}} 1^{f} \\
& \left(\text { where } \varphi_{23}\left(2^{c_{1}} 3^{d} 2^{e}\right)=3^{c_{2}} 2^{d_{1}} 3^{e_{1}}\right) \\
\sim & 2^{a_{1}} 3^{b_{2}} 1^{c_{3}} 2^{d_{1}} 1^{e_{2}} 3^{f_{1}} \\
& \left(\text { where } \varphi_{13}\left(1^{b_{1}} 3^{c_{2}}\right)=3^{b_{2}} 1^{c_{3}}, \text { etc. }\right) \\
\sim & 2^{a_{1}} 3^{b_{2}} 2^{c_{4}} 1^{d_{2}} 2^{e_{3}} 3^{f_{1}} \\
\sim & 3^{a_{2}} 2^{b_{3}} 3^{c_{5}} 1^{d_{2}} 2^{e_{3}} 3^{f_{1}} \\
\sim & 3^{a_{2}} 2^{b_{3}} 1^{c_{6}} 3^{d_{3}} 2^{e_{3}} 3^{f_{1}} \\
\sim & 3^{a_{2}} 2^{b_{3}} 1^{c_{6}} 2^{d_{4}} 3^{e_{4}} 2^{f_{2}} \\
\sim & 3^{a_{2}} 1^{b_{4}} 2^{c_{7}} 1^{d_{5}} 3^{e_{4}} 2^{f_{2}} \\
\sim & 1^{a_{3}} 3^{b_{5}} 2^{c_{7}} 3^{d_{6}} 1^{e_{5}} 2^{f_{2}} \\
\sim & 1^{a_{3}} 2^{b_{6}} 3^{c_{8}} 2^{d_{7}} 1^{e_{5}} 2^{f_{2}} \\
\sim & 1^{a_{3}} 2^{b_{6}} 3^{c_{8}} 1^{d_{8}} 2^{e_{6}} 1^{f_{3}} \\
& \sim 1^{a_{3}} 2^{b_{6}} 1^{c_{9}} 3^{d_{9}} 2^{e_{6}} 1^{f_{3}}
\end{aligned}
$$

Since this word has length $6,2^{6}$ checks must be performed to prove a particular blueprint realizes the $A_{3}(2)$ building. From our results we obtained:

Theorem 1. The only blueprints realizing the $A_{3}(2)$ building are relabelings of the natural blueprint for $A_{3}(2)$.

This theorem is proved by exhaustive computer search. Given our basic algorithm this case required roughly 120 hours of computer time on a
Silicon Graphics Indy computer, and the computer produced exactly the 8 blueprints that are relabelings of the natural blueprint.

From this theorem we obtain:

Corollary 9. Let $\Delta$ be a simply-laced building. Suppose the Coxeter diagram of $\Delta$ is connected, contains at least 4 vertices, and contains no triangles. If every panel of $\Delta$ lies on exactly 3 chambers (the $p=2$ case), and $\Delta$ conforms to a blueprint $\mathcal{B}=\left\{\varphi_{i, j}\right\}$, then all $\varphi_{i, j}$ are natural up to possible relabelings of the $S_{i}$ 's.

Proof. If the vertices $i$ and $j$ in the Coxeter diagram are connected, then there is a vertex $k$ such that $i, j$, and $k$ form an $A_{3}$ diagram. Hence by Theorem $1, \varphi_{i, j}$ is natural up to a relabeling. Moreover, if $k$ and $k^{\prime}$ are both adjacent to $i$, then the relabeling of $i$ must be the same since $i, k$, and $k^{\prime}$ form an $A_{3}$-subdiagram. Next, suppose $i$ and $j$ are not connected. The vertices $i$ and $j$ are connected by a path of length $m$. Inducting on $m$; if $m=2$, then $i$ and $j$ are part of an $A_{3}$ subdiagram and Theorem 1 yields the result. Else, consider the vertex $k$ adjacent to $i$ at distance $m-1$ from $j$. Then $i, j$, and $k$ form an $A_{1} \times A_{2}$ subdiagram. Moreover, we know that $\varphi_{i, k}$ and $\varphi_{j, k}$ are natural by the induction hypothesis. It remains to show that $\varphi_{i, j}$ is natural. Without loss of generality, assume $i=1, k=2$, and $j=3$ and consider the labeled word $1^{a} 2^{b} 1^{c} 3^{d}$. Then, $2^{a} 3^{d} \simeq 3^{d} 2^{a}$ since the $\varphi_{2,3}$ blueprint is natural. Similarly, $1^{a} 2^{b} 1^{c} \simeq 2^{a} 1^{a c-b} 2^{a}$ since the $\varphi_{1,2}$ blueprint is natural. Hence we obtain the following chain of elementary equivalences:

$$
\begin{aligned}
1^{a} 2^{b} 1^{c} 3^{d} & \simeq 2^{c} 1^{a c-b} 2^{a} 3^{d} \\
& \simeq 2^{c} 1^{b^{\prime}} 3^{d} 2^{a} \quad\left(b^{\prime}=a c-b\right) \\
& \simeq 2^{c} 3^{d^{\prime}} 1^{b^{\prime \prime}} 2^{a} \\
& \simeq 3^{d^{\prime}} 2^{a} 1^{b^{\prime \prime}} 2^{c} \\
& \simeq 3^{d^{\prime}} 1^{a} 2^{b^{\prime \prime \prime}} 1^{c} \quad\left(b^{\prime \prime \prime}=a c-b^{\prime \prime}\right) \\
& \simeq 1^{a^{\prime}} 3^{d^{\prime \prime}} 2^{b^{\prime \prime \prime}} 1^{c} \\
& \simeq 1^{a^{\prime}} 2^{b^{\prime \prime \prime}} 3^{d^{\prime \prime}} 1^{c} \\
& \simeq 1^{a^{\prime}} 2^{b^{\prime \prime \prime}} 1^{c^{\prime}} 3^{d^{\prime \prime \prime}} .
\end{aligned}
$$


Since we have a blueprint, by [Ronan 1989, Theorem 7.2] $a=a^{\prime}, b=b^{\prime \prime \prime}, c=c^{\prime}$, and $d=d^{\prime \prime \prime}$. Since $c^{\prime}=c$ and $d^{\prime \prime \prime}=d$, equation (3-3) implies for all $a$ that $1^{a} 3^{x} \simeq 3^{y} 1^{a}$. In turn we obtain $b^{\prime}=b^{\prime \prime}$ from equation (3-1). Let $b=a c-a$. Since $b^{\prime}=a$, by equation $(3-1) 1^{a} 3^{d} \simeq 3^{d^{\prime}} 1^{a}$. Now in equation (3-2) we have $3^{d^{\prime}} 1^{a} \simeq 1^{a} 3^{d^{\prime \prime}}$. This implies $d=d^{\prime \prime}$. But then the last line implies $1^{c} 3^{d} \simeq 3^{d} 1^{c}$. Since $c$ and $d$ are free, we obtain that $\varphi_{1,3}$ is natural. This completes the proof that $\mathcal{B}$ must be a relabeling of a natural blueprint.

As a Corollary to this we obtain Theorem 2.

Proof of Theorem 2. By the above, if there exists a simply-laced affine building over $\mathbb{Q}_{2}$ of rank at least 4 (with connected diagram) conforming to a blueprint then this buildings would conform to the natural blueprint. From [Ronan and Tits 1987, Example 6.1], we know that these blueprints realize the affine buildings over $\mathbb{F}_{2}((t))$. Hence they do not realize the $\mathbb{Q}_{2}$ buildings.

One result of this last corollary is that to use the idea of blueprints to construct $\mathbb{Q}_{2}$ buildings requires some generalization of the idea as was done in [Abramson $\geq 1998$ ]. An interesting question raised by this is whether the lack of such blueprints is due to the smallness of 2 or the restrictions placed on a blueprint by the building.

\section{REFERENCES}

[Abramson $\geq 1998$ ] M. Abramson, "Generalized blueprints and $p$-adic buildings I". To appear in Geometriae Dedicata.

[Kantor 1986] W. M. Kantor, "Generalized polygons, SCABs and GABs", pp. 79-158 in Buildings and the geometry of diagrams (Como, 1984), edited by L. A. Rosati, Lecture Notes in Math. 1181, Springer, Berlin, 1986.
[Köhler et al. 1984] P. Köhler, T. Meixner, and M. Wester, "Triangle groups", Comm. Algebra 12:13-14 (1984), 1595-1625.

[Köhler et al. 1985] P. Köhler, T. Meixner, and M. Wester, "The 2-adic affine building of type $\tilde{A}_{2}$ and its finite projections", J. Combin. Theory Ser. A 38:2 (1985), 203-209.

[Ronan 1984] M. A. Ronan, "Triangle geometries", $J$. Combin. Theory Ser. A 37:3 (1984), 294-319.

[Ronan 1989] M. Ronan, Lectures on buildings, Perspectives in Mathematics 7, Academic Press Inc., Boston, MA, 1989.

[Ronan and Tits 1987] M. A. Ronan and J. Tits, "Building buildings", Math. Ann. 278:1-4 (1987), 291-306.

[Rosen 1991] K. Rosen, Discrete mathematics and its applications, 2nd ed., McGraw-Hill, New York, 1991.

[Tits 1955a] J. Tits, "Groupes semi-simples complexes et géométrie projective", pp. Exp. No. 112, 115-125 in Séminaire Bourbaki, Vol. 3, Soc. Math. France, Paris, 1955.

[Tits 1955b] J. Tits, "Sur certaines classes d'espaces homogènes de groupes de Lie", Acad. Roy. Belg. Cl. Sci. Mém. 29:3 (1955), 268.

[Tits 1956] J. Tits, "Les groupes de Lie exceptionnels et leur interprétation géométrique", Bull. Soc. Math. Belg. 8 (1956), 48-81.

[Tits 1974] J. Tits, Buildings of spherical type and finite BN-pairs, Lecture Notes in Mathematics 386, Springer, Berlin, 1974.

[Tits 1981] J. Tits, "A local approach to buildings", pp. 519-547 in The geometric vein: the Coxeter Festschrift, edited by C. Davis et al., Springer, New York, 1981.

[Tits 1990] J. Tits, "Spheres of radius 2 in triangle buildings, I", pp. 17-28 in Finite geometries, buildings, and related topics (Pingree Park, CO, 1988), Oxford Sci. Publ., Oxford Univ. Press, New York, 1990. 
Michael Abramson, Department of Mathematics, University of Toledo, Toledo, OH (mabrams@newton.utoledo.edu)

Curtis D. Bennett, Department of Mathematics and Statistics, Bowling Green State University, Bowling Green, OH, 43403 (cbennet@math.bgsu.edu, http://www-math.bgsu.edu/ cbennet/)

Received January 7, 1998; accepted May 12, 1998 